\title{
DUALITY BETWEEN QUANTUM SYMMETRIC ALGEBRAS
}

\author{
XIAO-WU CHEN
}

\author{
Department of Mathematics \\ University of Science and Technology of China \\ Hefei 230026, Anhui, P. R. China
}

\begin{abstract}
Using certain pairings of couples, we obtain a large class of two-sided non-degenerated graded Hopf pairings for quantum symmetric algebras.
\end{abstract}

\section{INTRODUCTION}

One can construct three kinds of important graded Hopf algebras $T_{H}(M)$, $\operatorname{Cot}_{H}(M)$ and $S_{H}(M)$ for a given Hopf algebra $H$ and an $H$-Hopf bimodule $M$, which will be, respectively, referred as the tensor Hopf algebra, the cotensor Hopf algebra and the quantum symmetric algebra associated to the given couple $(H, M)$. These constructions go back to Nichols $[\mathrm{N}]$, and they are highlighted in Rosso's paper [Ro], who proves that the non-negative part of the quantum enveloping algebra $U_{\bar{q}}^{\geq 0}(\mathfrak{g})$ ( $q$ not a root of unity) of a complex semisimple Lie algebra $\mathfrak{g}$ is a quantum symmetric algebra.

A special case of the above Hopf algebras is of particular interest to representationists. If the Hopf algebra $H \simeq K \times \cdots \times K$ as algebras, then $T_{H}(M)$ is a path algebra of some quiver, and so the quantum symmetric algebra $S_{H}(M)$ is also a quotient of the path algebra (e.g., see [C], [CR1] and [GS]). Dually, if the Hopf algebra $H$ is group-like (not necessarily of finite dimension), then $\operatorname{Cot}_{H}(M)$ is a path coalgebra $[\mathrm{CM}]$ of some quiver, and hence $S_{H}(M)$ is a large subcoalgebra of the path coalgebra. Note that both of the above observations will lead to the concept of Hopf quivers by Cibils and Rosso [CR2]. In fact, these quiver presentations of the above Hopf algebras are very useful to study certain Hopf algebras, see [CHYZ] and [OZ], and they also could be used to classify some comodules of quantum groups, see [CZ].

E-mail: xwchen@mail.ustc.edu.cn.

Key words: Quantum symmetric algebras, Universal properties, Hopf pairings.

Mathematics Subject Classification 2000: 17B37,16W30.

Supported by the National Natural Science Foundation of China (Grant No. 10301033 and No. 10501041). 
Inspired by the above cited works, we study the three kinds of graded Hopf algebras and their universal properties. Moreover, using their universal properties, we can build up a large class of graded Hopf pairings for the quantum symmetric algebras, which can be seen as a generalization of a result by Nichols [N, Proposition 2.2.1] and the self-duality of $U_{q}^{\geq 0}(\mathfrak{g})$ (and its variants) via Rosso's isomorphism [Ro, Theorem 15] (and the remarks thereafter).

The paper is organized as follows: section 2 is devoted to recall the three known constructions of graded Hopf algebras from a given couple. We also include their universal properties. In section 3 , we prove the main results of this paper: Theorem 3.1 and Theorem 3.2, which claim that there exists graded Hopf pairings between certain quantum symmetric algebras, and furthermore, the existence of two-sided non-degenerated Hopf pairings characterizes quantum symmetric algebras. Note that the proof uses the technical notion of algebra-coalgebra pairings. As a special case, the self-duality of Hopf algebras is briefly discussed in 3.7.

All algebras and coalgebras will be over a fixed field $K$, and $\otimes$ means $\otimes_{K}$. Graded algebras (resp. coalgebras ...) will always mean positively-graded algebras (resp. coalgebras …) .

\section{Three CONSTRUCtions of GRAded Hopf Algebras}

This section is devoted to fix some notation and to recall three constructions of graded Hopf algebras from a given couple.

2.1. Let us recall some basic definitions in Hopf algebras (see $[\mathrm{Sw}]$ and $[\mathrm{M}]$ ). Throughout $H$ will be a Hopf algebra with comultiplication $\Delta_{H}$, counit $\varepsilon_{H}$ and antipode $S_{H}$. Sometimes we denote the multiplication of $H$ by $m_{H}$ and the unit of $H$ by $1_{H}$.

An $H$-Hopf bimodule $M$ is an $H$-bimodule (with the $H$-actions denoted by ".") and $H$-bicomodule with structure maps $\rho_{l}: M \longrightarrow H \otimes M$ and $\rho_{r}: M \longrightarrow M \otimes H$ such that

$$
\begin{aligned}
& \rho_{l}\left(h . m \cdot h^{\prime}\right)=\sum h_{1} m_{-1} h_{1}^{\prime} \otimes h_{2} \cdot m_{0} \cdot h_{2}^{\prime} \\
& \rho_{r}\left(h . m . h^{\prime}\right)=\sum h_{1} \cdot m_{0} \cdot h_{1}^{\prime} \otimes h_{2} m_{1} h_{2}^{\prime},
\end{aligned}
$$

where $h, h^{\prime} \in H$ and $m \in M$, and we use the Sweedler notation, i.e., $\Delta(h)=$ $\sum h_{1} \otimes h_{2}, \rho_{l}(m)=\sum m_{-1} \otimes m_{0}$ and $\rho_{r}(m)=\sum m_{0} \otimes m_{1}$ (e.g., see [Sw, p.10 and p.32]). For example, $H$ itself is an $H$-Hopf bimodule with the regular bimodule structure and the bicomodule structure maps $\rho_{l}=\rho_{r}=\Delta_{H}$.

We will refer to the above pair $(H, M)$ as a couple throughout this paper, where $H$ is a Hopf algebra and $M$ an $H$-Hopf bimodule. 
2.2. A Hopf algebra $H$ is said to be graded, if there exists a decomposition of vector spaces $H=\oplus_{n \geq 0} H_{n}$ such that

$$
\begin{gathered}
H_{n} H_{m} \subseteq H_{n+m}, \quad 1_{H} \in H_{0}, \quad S_{H}\left(H_{n}\right) \subseteq H_{n}, \\
\Delta_{H}\left(H_{n}\right) \subseteq \sum_{i+j=n} H_{i} \otimes H_{j}, \quad \varepsilon_{H}\left(H_{n}\right)=0 \quad \text { if } n>0,
\end{gathered}
$$

where $n, m \geq 0$.

Consider a graded Hopf algebra $H$. It is clear that $H_{0}$ is a subHopf algebra. Moreover, $H_{1}$ is an $H_{0}$-bimodule induced by the multiplication inside $H$. Note that

$$
\Delta_{H}\left(H_{1}\right) \subseteq H_{0} \otimes H_{1} \oplus H_{1} \otimes H_{0},
$$

thus there exist unique maps $\rho_{l}: H_{1} \longrightarrow H_{0} \otimes H_{1}$ and $\rho_{r}: H_{1} \longrightarrow H_{1} \otimes H_{0}$ such that

$$
\Delta_{H}(m)=\rho_{l}(m)+\rho_{r}(m), \quad \forall m \in H_{1} .
$$

The following result is well known and it can be easily checked.

Lemma 2.1. Use the notation above. Then $H_{1}$ is an $H_{0}$-Hopf bimodule with the structure maps $\rho_{l}$ and $\rho_{r}$, and thus $\left(H_{0}, H_{1}\right)$ is a couple.

We will say that the above couple $\left(H_{0}, H_{1}\right)$ is associated to the graded Hopf algebra $H$.

2.3. We will recall the constructions of graded Hopf algebras $T_{H}(M), \operatorname{Cot}_{H}(M)$ and $S_{H}(M)$ from a given couple $(H, M)$.

2.3.1. $T_{H}(M)$. As in 2.1, $H$ is a Hopf algebra and $M$ an $H$-Hopf bimodule. Denote $T_{H}(M)$ the tensor algebra associated to the $H$-bimodule $M$, i.e.,

$$
T_{H}(M)=H \oplus M \oplus\left(M \otimes_{H} M\right) \oplus \cdots \oplus M^{\otimes_{H} n} \oplus \cdots,
$$

where $M^{\otimes_{H} n}=M^{\otimes_{H} n-1} \otimes_{H} M$ for $n \geq 1$.

To avoid confusion, we will write $T_{H}(M) \underline{\otimes} T_{H}(M)$ for $T_{H}(M) \otimes T_{H}(M)$. Consider the following two maps

$$
\Delta_{H}: H \longrightarrow H \underline{\otimes} H \quad \subseteq T_{H}(M) \underline{\otimes} T_{H}(M)
$$

and

$$
\rho_{l} \oplus \rho_{r}: M \longrightarrow H \underline{\otimes} M+M \underline{\otimes} H \quad \subseteq T_{H}(M) \underline{\otimes} T_{H}(M) .
$$

It is direct to see that $T_{H}(M) \otimes T_{H}(M)$ is an $H$-bimodule via the algebra map $\Delta_{H}$ and the map $\rho_{l} \oplus \rho_{r}$ is an $H$-bimodule morphism. Applying the universal property of the tensor algebra $T_{H}(M)$ (e.g., see [N, Proposition 1.4.1]), we obtain that there exists a unique algebra map

$$
\delta: T_{H}(M) \longrightarrow T_{H}(M) \underline{\otimes} T_{H}(M)
$$

such that $\left.\delta\right|_{H}=\Delta_{H}$ and $\left.\delta\right|_{M}=\rho_{l} \oplus \rho_{r}$.

Using a similar argument, we obtain a unique algebra map

$$
\epsilon: T_{H}(M) \longrightarrow K
$$


such that $\left.\epsilon\right|_{H}=\varepsilon_{H}$ and $\left.\epsilon\right|_{M}=0$.

It is not hard to see that $\left(T_{H}(M), \delta, \epsilon\right)$ is a coalgebra, and thus $T_{H}(M)$ becomes a (graded) bialgebra. By [N, Proposition 1.5.1] or [M, Lemma 5.2.10], the bialgebra $T_{H}(M)$ is a Hopf algebra. In fact, one can describe the antipode more explicitly. Let $s_{1}: M \longrightarrow M$ be a map such that

$$
s_{1}(m)=-\sum S_{H}\left(m_{-1}\right) \cdot m_{0} \cdot S_{H}\left(m_{1}\right),
$$

where $\left(\operatorname{Id}_{H} \otimes \rho_{r}\right) \rho_{l}(m)=\sum m_{-1} \otimes m_{0} \otimes m_{1}$ and $m \in M$. Again by the universal property of the tensor algebra $T_{H}(M)$, there is a unique algebra map $s: T_{H}(M) \longrightarrow T_{H}(M)^{o p}$ such that $\left.s\right|_{H}=S_{H}$ and $\left.s\right|_{M}=s_{1}$, where $T_{H}(M)^{o p}$ is the opposite algebra of $T_{H}(M)$. One can deduce that the map $s$ is the antipode of $T_{H}(M)$ (here, one may use [Sw, p.73, Ex. 2) ]).

We will call the resulting Hopf algebra $T_{H}(M)$ the tensor Hopf algebra associated to the couple $(H, M)$.

We observe the following universal property of the tensor Hopf algebra $T_{H}(M)$. The proof follows immediately from the universal property of the tensor algebras and then the coalgebra structure of $T_{H}(M)$.

Proposition 2.2. Let $B=\oplus_{n>0} B_{n}$ be a graded Hopf algebra with the associated couple $\left(B_{0}, B_{1}\right)$. Then there exists a unique graded Hopf algebra morphism $\pi_{B}: T_{B_{0}}\left(B_{1}\right) \longrightarrow B$ such that the restriction of $\pi_{B}$ to $B_{0} \oplus B_{1}$ is the identity map.

Moreover, the map $\pi_{B}$ is surjective if and only if $B$ is generated by $B_{0}$ and $B_{1}$.

2.3.2. $\operatorname{Cot}_{H}(M)$. This is the dual construction of 2.3.1. As before, $(H, M)$ is a couple where $H$ is a Hopf algebra and $M$ an $H$-Hopf bimodule.

Denote $\operatorname{Cot}_{H}(M)$ the cotensor coalgebra with respect to the $H$-bicomodule $M$ (for details, see [N, p.1526], and [CHZ]), i.e.,

$$
\operatorname{Cot}_{H}(M)=H \oplus M \oplus\left(M \square_{H} M\right) \oplus \cdots \oplus M^{\square_{H} n} \oplus \cdots,
$$

where $M \square_{H} M$ is the cotensor product of $M$, and we denote $M^{\square_{H}}$ = $M \square_{H} M \square_{H} \cdots \square_{H} M$ (with $n$-copies of $M$ ). Note that $M^{\square_{H} n}$ is a subspace of $M^{\otimes n}$, and elements $\sum m^{1} \otimes \cdots \otimes m^{n} \in M^{\otimes n}$ which belong to $M^{\square_{H} n}$ will be written as $\sum m^{1} \square_{H} \cdots \square_{H} m^{n}$.

The coalgebra structure of $\operatorname{Cot}_{H}(M)$ is described as follows: the comultiplication $\Delta: \operatorname{Cot}_{H}(M) \longrightarrow \operatorname{Cot}_{H}(M) \otimes \operatorname{Cot}_{H}(M)$ is given by $\left.\Delta\right|_{H}=\Delta$, 
$\Delta(m)=\rho_{l}(m)+\rho_{r}(m)$ for all $m \in M$, and, in general,

$$
\begin{aligned}
\Delta\left(\sum m^{1} \square_{H} \cdots \square_{H} m^{n}\right) & =\sum\left(m^{1}\right)_{-1} \otimes\left(\left(m^{1}\right)_{0} \square_{H} \cdots \square_{H} m^{n}\right) \\
& +\sum_{i=1}^{n-1}\left(m^{1} \square_{H} \cdots \square_{H} m^{i}\right) \otimes\left(m^{i+1} \square_{H} \cdots \square m^{n}\right) \\
& +\sum\left(m^{1} \square \cdots \square_{H}\left(m^{n}\right)_{0}\right) \otimes\left(m^{n}\right)_{1} \\
& \in\left(H \otimes M^{\square_{H} n}\right) \\
& \bigoplus_{i=1}^{n-1}\left(M^{\square_{H}} \otimes M^{\square_{H}(n-i)}\right) \oplus\left(M^{\square_{H} n} \otimes H\right) \\
& \subseteq \operatorname{Cot}_{H} M \otimes \operatorname{Cot}_{H}(M),
\end{aligned}
$$

for any $\sum m^{1} \square_{H} \cdots \square_{H} m^{n} \in M^{\square n}$. The counit $\varepsilon: \operatorname{Cot}_{H}(M) \longrightarrow K$ is given by $\left.\varepsilon\right|_{H}=\varepsilon_{H}$ and $\left.\varepsilon\right|_{M^{\square}{ }^{n}}=0$ if $n \geq 1$.

Endow a bialgebra structure on $\operatorname{Cot}_{H}(M)$ as follows: by the universal property of the cotensor coalgebra $\operatorname{Cot}_{H}(M)$ (e.g., see [N, Proposition 1.4.2] or [CHZ, Lemma 3.2]), there exist unique graded coalgebra morphisms

$$
m: \operatorname{Cot}_{H}(M) \otimes \operatorname{Cot}_{H}(M) \longrightarrow \operatorname{Cot}_{H}(M) \text { and } e: K \longrightarrow \operatorname{Cot}_{H}(M),
$$

such that $\left.m\right|_{H \otimes H}$ is just the multiplication $m_{H}$ of the Hopf algebra $H$ and $\left.m\right|_{(H \otimes M) \oplus(M \otimes H)}$ is given by

$$
m\left(h \otimes n+n^{\prime} \otimes h^{\prime}\right)=h \cdot n+n^{\prime} \cdot h^{\prime}
$$

for all $n, n^{\prime} \in M$ and $h, h^{\prime} \in H$, and the unit map $e: K \longrightarrow \operatorname{Cot}_{H}(M)$ maps $1_{K}$ to $1_{H}$.

One can verify that $\left(\operatorname{Cot}_{H}(M), m, e\right)$ is an algebra, and thus $\operatorname{Cot}_{H}(M)$ becomes a (graded) bialgebra. By [N, Proposition 1.5.1] or [M, Lemma 5.2.10], the bialgebra $\operatorname{Cot}_{H}(M)$ is a graded Hopf algebra. Denote its antipode by $S$. It is not hard to see that

$$
\left.S\right|_{H}=S_{H} \quad \text { and } \quad S(m)=-\sum S_{H}\left(m_{-1}\right) \cdot m_{0} \cdot S_{H}\left(m_{1}\right)
$$

for all $m \in M$. In fact, by the universal property of the cotensor coalgebra $\operatorname{Cot}_{H}(M)$, the graded anti-coalgebra morphism $S$ is uniquely determined by the above two identities.

The resulting Hopf algebra $\left(\operatorname{Cot}_{H}(M), m, e, \Delta, \varepsilon, S\right)$ will be called the cotensor Hopf algebra associated to the couple $(H, M)$.

Recall that in a coalgebra $\left(C, \Delta_{C}, \varepsilon_{C}\right)$, the wedge is defined as $V \wedge_{C} W=$ $\Delta_{C}^{-1}(V \otimes C+C \otimes W)$ for any subspaces $V$ and $W$ of $C$. An important fact is that $H \wedge_{\operatorname{Cot}_{H}(M)} H=H \oplus M$. (To see this, first note that $H \oplus M \subseteq$ $H \wedge \wedge_{\operatorname{Cot}_{H}(M)} H$. Since $\operatorname{Cot}_{H}(M)$ is a graded coalgebra, then $H \wedge_{\operatorname{Cot}_{H}(M)} H$ is a graded subspace. Therefore, it suffices to show that

$$
\left(H \wedge \operatorname{Cot}_{H}(M) H\right) \cap M^{\square_{H} n}=0, \quad n \geq 2 .
$$


In fact, let $x \in\left(H \wedge_{\operatorname{Cot}_{H}(M)} H\right) \cap M^{\square_{H} n}$ be a nonzero element. Thus $\Delta(x) \in H \otimes M^{\square_{H} n}+M^{\square_{H} n} \otimes H$. Note that $x \in M^{\square_{H} n}$ and by the definition of the comultiplication $\Delta$, we know that the term belonging to $M \otimes M^{\square_{H} n-1}$ which occurs in $\Delta(x)$ is not zero. This is a contradiction.)

Dual to Proposition 2.2, we observe the following universal property of the cotensor Hopf algebra $\operatorname{Cot}_{H}(M)$. Note that it is a slight generalization of [OZ, Theorem 4.5] (the proof of the second statement needs to use the above recalled fact).

Proposition 2.3. Let $B=\oplus_{n \geq 0} B_{n}$ be a graded Hopf algebra with the associated couple $\left(B_{0}, B_{1}\right)$. Then there exists a unique graded Hopf algebra morphism $i_{B}: B \longrightarrow \operatorname{Cot}_{B_{0}}\left(B_{1}\right)$ such that its restriction to $B_{0} \oplus B_{1}$ is the identity map.

Moreover, the map $i_{B}$ is injective if and only if $B_{0} \wedge_{B} B_{0}=B_{0} \oplus B_{1}$.

2.3.3. $S_{H}(M)$. As above, $(H, M)$ is a couple. Denote $S_{H}(M)$ be the graded subalgebra of the cotensor Hopf algebra $\operatorname{Cot}_{H}(M)$ generated by $H$ and $M$. Clearly, $S_{H}(M)$ is a graded subHopf algebra of $\operatorname{Cot}_{H}(M)$. We will call $S_{H}(M)$ the quantum symmetric algebra (see [Ro, p.407]) associated to the couple $(H, M)$.

The following result is a direct consequence of Proposition 2.3.

Corollary 2.4. Let $B=\oplus_{n \geq 0} B_{n}$ be a graded Hopf algebra generated by $B_{0}$ and $B_{1}$. Denote $\left(B_{0}, B_{1}\right)$ the couple associated to $B$. Then there exists a unique graded Hopf algebra epimorphism $j_{B}: B \longrightarrow S_{B_{0}}\left(B_{1}\right)$ such that its restriction to $B_{0} \oplus B_{1}$ is the identity map.

Remark 2.5. (1). We may deduce Nichols's result [N, 2.2] from Proposition 2.3: the map $i_{B}$ in Proposition 2.3 is an isomorphism if and only if $B$ is generated by $B_{0}$ and $B_{1}$ and $B_{0} \wedge_{B} B_{0}=B_{0} \oplus B_{1}$. In particular, if $B_{0}$ is cosemisimple, we see that $i_{B}: B \simeq S_{B_{0}}\left(B_{1}\right)$ if and only if $B$ is coradicallygraded and generated by $B_{0}$ and $B_{1}$.

(2). As a special case of Corollary 2.4 , for every couple $(H, M)$, there is a unique graded Hopf algebra epimorphism

$$
\pi_{(H, M)}: T_{H}(M) \longrightarrow S_{H}(M)
$$

such that its restriction to $H \oplus M$ is the identity map, where we denote $j_{T_{H}(M)}$ by $\pi_{(H, M)}$. Denote the kernel of $\pi_{(H, M)}$ by $I(H, M)$, hence it is a graded Hopf ideal of $T_{H}(M)$ and $T_{H}(M) / I(H, M) \simeq S_{H}(M)$.

\section{Duality Between Quantum Symmetric Algebras}

3.1. Let us recall the definition of Hopf pairings (see $[\mathrm{K}], \mathrm{p} .110$ ). Let $H$ and $B$ be Hopf algebras. A Hopf pairing $\phi: H \times B \longrightarrow K$ is a bilinear map 
such that

$$
\begin{aligned}
& \phi\left(1_{H}, b\right)=\varepsilon_{B}(b) ; \quad \phi\left(h, 1_{B}\right)=\varepsilon_{H}(h) ; \\
& \phi(h, b c)=\sum \phi\left(h_{1}, b\right) \phi\left(h_{2}, c\right) ; \\
& \phi(h g, b)=\sum \phi\left(h, b_{1}\right) \phi\left(g, b_{2}\right) ; \\
& \phi\left(S_{H}(h), b\right)=\phi\left(h, S_{B}(b)\right),
\end{aligned}
$$

where $h, g \in H, b, c \in B$, and $S_{H}$ and $S_{B}$ are the antipodes of $H$ and $B$, respectively. Note that one can define the transpose $\phi^{t}: B \times H \longrightarrow K$ by $\phi^{t}(b, h)=\phi(h, b)$, which is also a Hopf pairing.

Assume further that $H=\oplus_{n \geq 0} H_{n}$ and $B=\oplus_{n \geq 0} B_{n}$ are graded Hopf algebras. A Hopf pairing $\phi: H \times B \longrightarrow K$ is said to be graded if $\phi\left(H_{i}, B_{j}\right)=$ 0 if $i \neq j$.

3.2. We introduce an analogous concept of Hopf pairings. Let $(H, M)$ and $(B, N)$ be couples. Denote the $H$-comodule (resp. $B$-comodule) structure on $M($ resp. $N)$ by $\rho_{l}$ and $\rho_{r}$ (resp. $\delta_{l}$ and $\left.\delta_{r}\right)$. Denote the actions by "." .

A pairing between the couples $(H, M)$ and $(B, N)$, denoted by

$$
\left(\phi_{0}, \phi_{1}\right):(H, M) \times(B, N) \longrightarrow K,
$$

is given by a Hopf pairing $\phi_{0}: H \times B \longrightarrow K$ and a bilinear map $\phi_{1}$ : $M \times N \longrightarrow K$ such that

$$
\begin{aligned}
\phi_{1}(h . m . g, n) & =\phi_{0}\left(h, n_{-1}\right) \phi_{1}\left(m, n_{0}\right) \phi_{0}\left(g, n_{1}\right), \\
\phi_{1}(m, \text { b.n.c }) & =\phi_{0}\left(m_{-1}, b\right) \phi_{1}\left(m_{0}, n\right) \phi_{0}\left(m_{1}, c\right),
\end{aligned}
$$

where $h, g \in H, b, c \in B, m \in M$ and $n \in N$, and $\left(\operatorname{Id}_{H} \otimes \rho_{r}\right) \rho_{l}(m)=$ $\sum m_{-1} \otimes m_{0} \otimes m_{1}$ and $\left(\operatorname{Id}_{B} \otimes \delta_{r}\right) \delta_{l}(n)=\sum n_{-1} \otimes n_{0} \otimes n_{1}$.

We have our main results.

Theorem 3.1. Let $\left(\phi_{0}, \phi_{1}\right):(H, M) \times(B, N) \longrightarrow K$ be a pairing between couples. Then there exists a unique graded Hopf pairing

$$
\phi: S_{H}(M) \times S_{B}(N) \longrightarrow K
$$

extending $\phi_{0}$ and $\phi_{1}$.

Moreover, $\phi$ is two-sided non-degenerated if and only if $\phi_{0}$ and $\phi_{1}$ are.

and

Theorem 3.2. Let $H=\oplus_{n \geq 0} H_{n}$ (resp. $B=\oplus_{n \geq 0} B_{n}$ ) be graded Hopf algebras generated by $H_{0}$ and $H_{1}$ (resp. $B_{0}$ and $B_{1}$ ). Assume that there exists a two-sided non-degenerated graded Hopf pairing $\psi: H \times B \longrightarrow K$. Then $j_{H}: H \simeq S_{H_{0}}\left(H_{1}\right)$ and $j_{B}: B \simeq S_{B_{0}}\left(B_{1}\right)$, where the maps $j_{H}$ and $j_{B}$ are explained in Corollary 2.4. 
3.3. To prove the above two results, we need to introduce the following technical concept, which is essentially the same as the (graded) duality between algebras and coalgebras.

Let $A$ be an algebra and $\left(C, \Delta_{C}, \varepsilon_{C}\right)$ a coalgebra. Let $\phi: A \times C \longrightarrow K$ be a bilinear map, and define $\phi^{*}: A \longrightarrow C^{*}$ by $\phi^{*}(a)(c)=\phi(a, c)$. We say that $\phi$ is an algebra-coagebra pairing if

$$
\phi\left(1_{A}, c\right)=\varepsilon_{C}(c) \text { and } \phi\left(a a^{\prime}, c\right)=\sum \phi\left(a, c_{1}\right) \phi\left(a, c_{2}\right),
$$

for all $a, a^{\prime} \in A$ and $c \in C$, where $1_{A} \in A$ is the identity element and $\Delta(c)=\sum c_{1} \otimes c_{2}$.

In fact, it is easily checked that $\phi$ is an algebra-coalgebra pairing if and only if $\phi^{*}$ is an algebra morphism, where $C^{*}$ is the dual algebra of the coalgebra $C$.

The graded version of the above concept is as follows: let $A=\oplus_{n \geq 0} A_{n}$ be a graded algebra and $C=\oplus_{n \geq 0} C_{n}$ a graded coalgebra, an algebra-coalgebra pairing $\phi: A \times C \longrightarrow K$ is said to be graded, if $\phi\left(A_{i}, C_{j}\right)=0$ for $i \neq j$. As above, we can define a graded map $\phi^{*}: A \longrightarrow C^{\text {gr }}$, where $C^{\text {gr }}=\oplus_{n \geq 0} C_{n}^{*}$ is the graded dual of $C$. One sees that $\phi$ is a graded algebra-coalgebra pairing if and only if $\phi^{*}$ is a graded algebra map.

In what follows, we assume that $A$ is an algebra and $M$ an $A$-bimodule (with actions denoted by "."), and $\left(C=\oplus_{n \geq 0} C_{n}, \Delta_{C}, \varepsilon_{C}\right)$ is a graded coalgebra. Clearly there exist unique maps

$$
\delta_{l}: C_{1} \longrightarrow C_{0} \otimes C_{1} \quad \text { and } \quad \delta_{r}: C_{1} \longrightarrow C_{1} \otimes C_{0}
$$

such that $\Delta_{C}(c)=\delta_{l}(c)+\delta_{r}(c)$ for all $c \in C_{1}$. By abuse of notation, write $\delta_{l}(c)=\sum c_{-1} \otimes c_{0}$ and $\delta_{r}(c)=\sum c_{0} \otimes c_{1}$. Thus $\Delta_{C}(c)=\sum c_{-1} \otimes c_{0}+$ $\sum c_{0} \otimes c_{1}$.

Assume further that $\phi_{0}: A \times C_{0} \longrightarrow K$ is an algebra-coalgebra pairing, and $\phi_{1}: M \times C_{1} \longrightarrow K$ is a bilinear map such that

$$
\phi_{1}\left(a . m \cdot a^{\prime}, c\right)=\sum \phi_{0}\left(a, c_{-1}\right) \phi_{1}\left(m, c_{0}\right) \phi_{0}\left(a^{\prime}, c_{1}\right)
$$

for all $a, a^{\prime} \in A$ and $c \in C_{1}$, where $\left(\operatorname{Id}_{C_{0}} \otimes \delta_{r}\right) \delta_{l}(c)=\sum c_{-1} \otimes c_{0} \otimes c_{1}$.

We have the following

Lemma 3.3. Assume that $\phi_{0}$ and $\phi_{1}$ are as above. There exists a unique graded algebra-coalgebra pairing $\phi: T_{A}(M) \times C \longrightarrow K$ extending $\phi_{0}$ and $\phi_{1}$.

Proof This is just a variant of the universal property of the tensor algebra $T_{A}(M)$. Using $\phi_{0}$ and $\phi_{1}$, we can define $\phi_{0}^{*}: A \longrightarrow C_{0}^{*} \subseteq C^{\text {gr }}$ and $\phi_{1}^{*}$ : $M \longrightarrow C_{1}^{*} \subseteq C^{\text {gr }}$. Note that $\phi_{0}^{*}$ is an algebra map and $\phi_{1}^{*}$ is an $A$-bimodule morphism (exactly by the condition (3.1)). 
Now by the universal property of the tensor algebra $T_{A}(M)$, there exists a unique graded algebra map

$$
\phi^{*}: T_{A}(M) \longrightarrow C^{\mathrm{gr}}
$$

extending $\phi_{0}^{*}$ and $\phi_{1}^{*}$. Define $\phi$ by $\phi(x, c)=\phi^{*}(x)(c)$, for all $x \in T_{A}(M)$ and $c \in C$. Immediately, $\phi$ is the unique graded algebra-coalgebra pairing extending $\phi_{0}$ and $\phi_{1}$. This completes the proof.

3.4. Recall that any pairing $\phi: A \times C \longrightarrow K$ is said to be left nondegenerated provided that for each nonzero $y \in C$ there is some $x \in A$ such that $\phi(x, y) \neq 0$. Let us go to the situation of Theorem 3.1 and 3.2: we are given a pairing of couples $\left(\phi_{0}, \phi_{1}\right):(H, M) \times(B, N) \longrightarrow K$. The following result is of independent interest.

Proposition 3.4. There exists a unique graded Hopf pairing

$$
\phi: T_{H}(M) \times \operatorname{Cot}_{B}(N) \longrightarrow K
$$

extending $\phi_{0}$ and $\phi_{1}$.

Moreover, if $\phi_{0}$ and $\phi_{1}$ are left non-degenerated, then so is $\phi$.

Proof By Lemma 3.3, there exists a unique graded algebra-coalgebra pairing $\phi: T_{H}(M) \times \operatorname{Cot}_{B}(N) \longrightarrow K$ extending $\phi_{0}$ and $\phi_{1}$. We will show that $\phi$ is a Hopf pairing.

Use the notation in 2.3.1 and 2.3.2. First we have $\phi\left(x, 1_{B}\right)=\epsilon(x)$ and $\phi\left(1_{H}, y\right)=\varepsilon(y)$ for all $x \in T_{H}(M), y \in \operatorname{Cot}_{B}(N)$. (To see this, since $\phi$ is graded, we have $\phi\left(x, 1_{B}\right)=0=\epsilon(x)$ for $x \in M^{\otimes_{H} n}, n \geq 1$; and for $x \in H$, $\phi\left(x, 1_{B}\right)=\phi_{0}\left(x, 1_{B}\right)=\epsilon(x)$. Similarly one obtains that $\phi\left(1_{H}, y\right)=\varepsilon(y)$.) Define two bilinear maps

$$
\Psi, \Phi: T_{H}(M) \times\left(\operatorname{Cot}_{B}(N) \otimes \operatorname{Cot}_{B}(N)\right) \longrightarrow K
$$

such that $\Psi(x, y \otimes z)=\phi(x, y z)$ and $\Phi(x, y \otimes z)=\sum \phi\left(x_{1}, y\right) \phi\left(x_{2}, z\right), x \in$ $T_{H}(M), y, z \in \operatorname{Cot}_{B}(N)$. Note that both $\Psi$ and $\Phi$ are graded algebracoalgebra pairings, and by the defining properties of the pairing $\left(\phi_{0}, \phi_{1}\right)$, we have

$$
\left.\Psi\right|_{H \times(B \otimes B)}=\left.\Phi\right|_{H \times(B \otimes B)} \quad \text { and }\left.\quad \Psi\right|_{M \times(B \otimes N+N \otimes B)}=\left.\Phi\right|_{M \times(B \otimes N+N \otimes B)} .
$$

Now by the uniqueness part of Lemma 3.3, we obtain that $\Psi=\Phi$, i.e., $\phi(x, y z)=\phi\left(x_{1}, y\right) \phi\left(x_{2}, z\right)$.

Similarly, we construct two graded algebra-coalgebra pairings

$$
\Psi^{\prime}, \Phi^{\prime}: T_{H}(M) \times \operatorname{Cot}_{B}(N)^{c o p} \longrightarrow K
$$

such that $\Psi^{\prime}(x, y)=\phi(s(x), y)$ and $\Phi^{\prime}(x, y)=\phi(x, S(y))$, where $x \in T_{H}(M)$ and $y \in \operatorname{Cot}_{B}(N)$, and $\operatorname{Cot}_{B}(N)^{c o p}$ denotes the opposite coalgebra. By a similar argument as above, we show that $\phi(s(x), y)=\phi(x, S(y))$.

Summing up the above, we have shown that $\phi$ is the unique required graded Hopf pairing. 
For the second statement, assume that $\phi_{0}$ and $\phi_{1}$ are left non-degenerated, we need to show that for every nonzero element $y \in N^{\square}{ }_{B}$, there exists some $x \in M^{\otimes_{H} i}$ such that $\phi(x, y) \neq 0, i \geq 2$. Since $\phi_{1}: M \times N \longrightarrow K$ is left nondegenerated, hence the following bilinear map will be left non-degenerated:

$$
\phi_{1}^{\otimes i}: M^{\otimes i} \times N^{\otimes i} \longrightarrow K,
$$

where $\phi_{1}^{\otimes i}\left(m^{1} \otimes \cdots \otimes m^{i}, n^{1} \otimes \cdots \otimes n^{i}\right)=\prod_{r=1}^{i} \phi_{1}\left(m^{r}, n^{r}\right)$. Note that $N^{\square_{B} i} \subseteq N^{\otimes i}$, hence for the nonzero $y \in N^{\square_{B} i}$, there exists some $x^{\prime} \in M^{\otimes i}$ such that $\phi_{1}^{\otimes i}\left(x^{\prime}, y\right) \neq 0$.

Denote by $p: M^{\otimes i} \longrightarrow M^{\otimes_{H} i}$ the natural projection map. By the fact that $\phi$ is an algebra-coalgebra pairing, we have

$$
\phi\left(p\left(x^{\prime}\right), y\right)=\phi_{1}^{\otimes n}\left(x^{\prime}, y\right) .
$$

Take $x=p\left(x^{\prime}\right)$. We see that $\phi(x, y) \neq 0$, finishing the proof.

3.5. Proof of Theorem 3.1: Consider the following composite of morphisms between graded Hopf algebras

$$
\pi: T_{B}(N) \stackrel{\pi_{(B, N)}}{\longrightarrow} S_{B}(N) \hookrightarrow \operatorname{Cot}_{B}(N),
$$

where the map $\pi_{(B, N)}$ is described in Remark 2.5(2) and the second map is just the inclusion. Applying Proposition 3.4, we have a graded Hopf pairing $\phi^{\prime}: T_{H}(M) \times \operatorname{Cot}_{B}(N) \longrightarrow K$ extending $\phi_{0}$ and $\phi_{1}$. Define

$$
\phi^{\prime \prime}: T_{H}(M) \times T_{B}(N) \longrightarrow K .
$$

by putting $\phi^{\prime \prime}(x, y)=\phi^{\prime}(x, \pi(y))$. Thus $\phi^{\prime \prime}$ is a graded Hopf pairing.

Note that $I(B, N)$ is the kernel of $\pi_{(B, N)}$ and thus the kernel of $\pi$, we see that

$$
\phi^{\prime \prime}\left(T_{H}(M), I(B, N)\right)=0 .
$$

We now claim that

$$
\phi^{\prime \prime}\left(I(H, M), T_{B}(N)\right)=0 .
$$

For this end, apply Proposition 3.4 again, we have a graded Hopf pairing

$$
\psi: \operatorname{Cot}_{H}(M) \times T_{B}(N) \longrightarrow K
$$

extending $\phi_{0}$ and $\phi_{1}$. Consider the following composite

$$
\pi^{\prime}: T_{H}(M) \stackrel{\pi_{(H, M)}}{\longrightarrow} S_{H}(M) \hookrightarrow \operatorname{Cot}_{H}(M) .
$$

Define $\psi^{\prime}: T_{H}(M) \times T_{B}(N) \longrightarrow K$ by $\psi^{\prime}(x, y)=\psi\left(\pi^{\prime}(x), y\right)$. Since $\pi^{\prime}$ is a (graded) Hopf algebra morphism, thus $\psi^{\prime}$ is a graded Hopf pairing. Similarly as above, we have $\psi^{\prime}\left(I(H, M), T_{B}(N)\right)=0$. Note that both $\phi^{\prime \prime}$ and $\psi^{\prime}$ are graded algebra-coalgebra pairings extending $\phi_{0}$ and $\phi_{1}$. Applying Lemma 3.3 , we have $\phi^{\prime \prime}=\psi^{\prime}$. This proves the claim. 
So we have shown that $\phi^{\prime \prime}\left(T_{H}(M), I(B, N)\right)=0$ and $\phi^{\prime \prime}\left(I(H, M), T_{B}(N)\right)=$ 0 . Recall from Remark 2.5(2) that we have

$$
T_{H}(M) / I(H, M) \simeq S_{H}(M) \quad \text { and } \quad T_{B}(N) / I(B, N) \simeq S_{B}(N) .
$$

Thus we deduce that $\phi^{\prime \prime}$ induces a unique graded Hopf pairing

$$
\phi: S_{H}(M) \times S_{B}(N) \longrightarrow K
$$

such that the following diagram commutes

$$
\begin{aligned}
T_{H}(M) \times T_{B}(N) \stackrel{\phi^{\prime \prime}}{\longrightarrow} K \\
\pi_{(H, M)} \times \pi_{(B, N)} \downarrow \\
S_{H}(M) \times S_{B}(N) \stackrel{\phi}{\longrightarrow} K .
\end{aligned}
$$

Explicitly, $\phi\left(\pi_{(H, M)}(x), \pi_{(B, N)}(y)\right)=\phi^{\prime \prime}(x, y)$, for all $x \in T_{H}(M)$ and $y \in T_{B}(N)$.

Obviously, the pairing $\phi$ extends the maps $\phi_{0}$ and $\phi_{1}$, as required. Note that the uniqueness of $\phi$ is trivial, since $S_{H}(M)$, as an algebra, is generated by $H$ and $M$. (Here, one needs to consult the fourth identity in the definition of Hopf pairing, see 3.1).

For the second statement, assume that $\phi_{0}$ and $\phi_{1}$ are two-sided nondegenerated. By Proposition 3.4, we have that $\phi^{\prime}$ is left non-degenerated. Note that $\phi\left(\pi_{(H, M)}(x), \pi_{(B, N)}(y)\right)=\phi^{\prime \prime}(x, y)=\phi^{\prime}(x, \pi(y))$. This implies that $\phi$ is left non-degenerated. For right non-degeneratedness, first apply Proposition 3.4 to $\psi^{t}$ (the transpose of $\psi$, see 3.1 ), we deduce that $\psi^{t}$ is left non-degenerated, that is, $\psi$ is right non-degenerated. Now note that $\phi\left(\pi_{(H, M)}(x), \pi_{(B, N)}(y)\right)=\phi^{\prime \prime}(x, y)=\psi^{\prime}(x, y)=\psi\left(\pi^{\prime}(x), y\right)$, which implies that $\phi$ is right non-degenerated. This completes the proof.

3.6. Proof of Theorem 3.2: $\quad$ Since the Hopf pairing $\psi: H \times B \longrightarrow K$ is two-sided non-degenerated, so are the restrictions $\phi_{0}:=\left.\psi\right|_{H_{0} \times B_{0}}$ and $\phi_{1}:=\left.\psi\right|_{H_{1} \times B_{1}}$. Now applying Theorem 3.1, there exists a unique graded Hopf pairing $\phi: S_{H_{0}}\left(H_{1}\right) \times S_{B_{0}}\left(B_{1}\right) \longrightarrow K$ extending $\phi_{0}$ and $\phi_{1}$.

We claim that the following diagram commutes

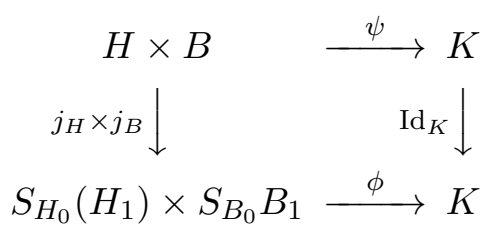

where the maps $j_{H}$ and $j_{B}$ are explained in Corollary 2.4.

To see this, set $\psi^{\prime}=\phi \circ\left(j_{H} \times j_{B}\right)$. Thus both $\psi$ and $\psi^{\prime}$ are graded Hopf pairings. Note that

$$
\left.\psi\right|_{H_{0} \times B_{0}}=\left.\psi^{\prime}\right|_{H_{0} \times B_{0}} \text { and }\left.\quad \psi\right|_{H_{1} \times B_{1}}=\left.\psi^{\prime}\right|_{H_{1} \times B_{1}} .
$$


Since $H$ is generated by $H_{0}$ and $H_{1}$, it follows from the fourth identity in the definition of Hopf pairing (see 3.1) that $\psi=\psi^{\prime}$. This shows the claim.

By Corollary 2.4, the maps $j_{H}$ and $j_{B}$ are epimorphisms. The fact that both $\phi$ and $\psi$ are two-sided non-degenerated immediately implies that $j_{H}$ : $H \simeq S_{H_{0}}\left(H_{1}\right)$ and $j_{B}: B \simeq S_{B_{0}}\left(B_{1}\right)$. This completes the proof.

3.7. Self-dual couples. We end our paper with a special case of Theorem 3.1 , which is of independent interest.

Recall that a Hopf algebra $H$ is said to be self-dual, if there exists a twosided non-degenerated Hopf pairing $\phi: H \times H \longrightarrow K$. Similarly, a graded Hopf algebra $H=\oplus_{n \geq 0} H_{n}$ is said to be graded self-dual, if the Hopf pairing $\phi$ is graded.

A couple $(\mathrm{H}, \mathrm{M})$, where $H$ is a Hopf algebra and $M$ an $H$-Hopf bimodule, is said to be self-dual, if there exists a pairing

$$
\left(\phi_{0}, \phi_{1}\right):(H, M) \times(H, M) \longrightarrow K
$$

such that both $\phi_{0}$ and $\phi_{1}$ are two-sided non-degenerated. Note that in this case, the $H$-Hopf bimodule $M$ is exactly the self-dual Hopf bimodule in $[\mathrm{GM}]$ and $[\mathrm{HLY}]$.

The following result is a direct consequence of Theorem 3.1.

Corollary 3.5. Let $(H, M)$ be a couple as above. Then the quantum symmetric algebra $S_{H}(M)$ is graded self-dual if and only if the couple $(H, M)$ is self-dual.

\section{REFERENCES}

[CHYZ] X. W. Chen, H. L. Huang, Y. Ye, and P. Zhang, Monomial Hopf algebras, J. Algebra 275(2004), 212-232.

[CHZ] X. W. Chen, H. L. Huang, and P. Zhang, Dual Gabriel theorem with applications, Sci. in China, Ser. A Math. 49(1)(2006), 9-26.

[CZ] X. W. Chen and P. Zhang, Comodules of $U_{q}\left(s l_{2}\right)$ and modules of $S L_{q}(2)$ via quiver methods, J. Pure Appl. Algebra, to appear.

[CM] W. Chin and S. Montgomery, Basic coalgebras, In: Modular interfaces (Reverside, CA, 1995), 41-47, AMS/IP Stud. Adv. Math. 4, Amer. Math. Soc., Providence, RI, 1997.

[C] C. Cibils, A quiver quantum group, Comm. Math. Phys. 157 (1993), 459-477.

[CR1] C. Cibils and M. Rosso, Algebres des chemins quantique, Adv. Math. 125 (1997), 171-199.

[CR2] C. Cibils and M. Rosso, Hopf quivers, J. Algebra 254(2002), 241-251.

[GM] E. L. Green and E. N. Marcos, Self-dual Hopf algebras, Comm. Algebra 28 (6) (2000), 2735-2744.

[GS] E. L. Green and Ø. Solberg, Basic Hopf algebras and quantum groups, Math. Z. 229(1998), 45-76. 
[HLY] H. L. Huang, L. B. Li, and Y. Ye, Self-dual Hopf quivers, Comm. Algebra 33 (12) (2005), 4505-4514

[K] C. Kassel, Quantum Groups. Graduate Texts in Math. 155, Springer-Verlag, New York, 1995.

[M] S. Montgomery, Hopf Algebras and Their Actions on Rings. CBMS Regional Conf. Series in Math. 82, Amer. Math. Soc., Providence, RI, 1993.

[N] W. Nichols, Bialgebra of type I, Comm. Algebra 15 (1978), 1521-1552.

[OZ] F. van Oystaeyen and P. Zhang, Quiver Hopf algebras, J. Algebra 280(2004), 577-589.

[Ro] M. Rosso, Quantum groups and quantum shuffles, Invent. Math. 133 (1998), 339416.

[Sw] M. E. Sweedler, Hopf Algebras, Benjamin, New York, 1969. 